\title{
EL INTERNADO RURAL COMO ACERCAMIENTO A LA ATENCIÓN PRIMARIA EN SALUD, LA EXPERIENCIA DE UNA UNIVERSIDAD DE CUSCO, PERÚ
}

\section{RURAL INTERNSHIP AS AN APPROACH TO PRIMARY HEALTH CARE -THE EXPERIENCE OF A UNIVERSITY IN CUSCO, PERU}

\author{
Roy R. Vásquez-Sullca',a, Santiago Saco-Méndez²,b, \\ César J. Pereira-Victorio ${ }^{3,4, b}$
}

Sr. Editor. La Organización Panamericana de la Salud, dentro del marco de la "Renovación de la Atención primaria de la salud en las Américas", lanzó en el 2008 la "Formación en Medicina orientada hacia la atención primaria de la salud", documento donde se señala la necesidad de trabajar en la formación de los médicos de la región, fortaleciendo sus competencias en atención primaria en salud (APS) ${ }^{(1)}$. Sin embargo, existen pocos escenarios donde estas premisas se cumplen de manera práctica. En Latinoamérica, dos universidades de Perú y una de Chile han implementado en su plan curricular el internado rural ( $\operatorname{lnR})$, entendido este como el ejercicio pre-profesional de estudiantes de ciencias de la salud en actividades de medicina integral en establecimientos de salud en comunidades rurales bajo la supervisión de tutores-docentes.

En el Perú, el InR de la Universidad Nacional de San Antonio Abad del Cusco (UNSAAC) se realiza en el sexto año de formación, tiene una duración de doce semanas y cuenta con nueve sedes de atención primaria (SAP). En la Universidad Peruana Cayetano Heredia tiene una duración de 10 semanas, se realiza en el séptimo año de formación en seis SAP ${ }^{(2)}$, y en la Universidad de Chile tiene una duración de cuatro semanas y cuenta con dos SAP ${ }^{(3)}$. Las tres universidades tienen el mismo objetivo: reforzar los conocimientos y realizar acciones de promoción, prevención, recuperación y rehabilitación de la salud. En Perú, los internos participan, además,

\footnotetext{
Asociación Científica de Estudiantes de Medicina Humana, Universidad Nacional de San Antonio Abad del Cusco. Cusco, Perú.

Facultad de Medicina, Universidad Nacional de San Antonio Abad del Cusco. Cusco, Perú.

Complejo Hospitalario Universitario de Albacete. Albacete, España.

Universidad Complutense de Madrid. Madrid España.

Estudiante de Medicina Humana; ${ }^{\mathrm{b}}$ médico cirujano, máster en Salud Pública.

Recibido: 28-01-13 Aprobado: 06-02-13
}

Citar como: Vásquez-Sullca RR, Saco-Méndez S, Pereira-Victorio CJ. El internado rural como acercamiento a la atención primaria en salud, la experiencia de una universidad de Cusco, Perú [carta]. Rev Peru Med Exp Salud Publica. 2013;30(2):344-5.
Tabla 1. Centros de salud sedes del internado rural de la UNSAAC

\begin{tabular}{llccrc}
\hline Provincia & $\begin{array}{c}\text { Centros } \\
\text { de salud }\end{array}$ & $\begin{array}{c}\text { Nivel } \\
\text { puintil } \\
\text { de } \\
\text { pobreza }\end{array}$ & & Población & $\begin{array}{c}\text { Altitud * } \\
\text { (metros) }\end{array}$ \\
\hline Acomayo & Acomayo & I-4 & 1 & 5380 & 3221 \\
\hline Acomayo & Pomacanchi & I-4 & 1 & 8340 & 3693 \\
\hline Paucartambo Paucartambo & I-4 & 1 & 12057 & 3005 \\
\hline Paucartambo Pillcopata & I-4 & 1 & 4790 & 689 \\
\hline Paucartambo Huancarani & I-4 & 1 & 6910 & 3850 \\
\hline Quispicanchi & Ccatcca & I-4 & 1 & 14346 & 3700 \\
Quispicanchi & Ocongate & I-4 & 1 & 13578 & 3540 \\
Quispicanchi & Quiquijana & I-4 & 1 & 10340 & 3553 \\
\hline Urubamba & Urubamba & I-4 & 2 & 17787 & 2869 \\
\hline
\end{tabular}

UNSAAC: Universidad Nacional de San Antonio Abad del Cusco Fuente: Mapa de pobreza distrital de FONCODES 2006, con indicadores actualizados con el censo del 2007, * Directorio nacional de municipalidades provinciales, distritales y de centros poblados 2012 Instituto Nacional de Estadística e Informática

activamente de los programas de salud y de las estrategias sanitarias nacionales, actividades que se consideran serán de ayuda para su posterior ejercicio profesional.

En la UNSAAC, el InR se instauró el año de 1988, se creó con la finalidad de enriquecer la formación de los estudiantes de ciencias de la salud y reforzar sus conocimientos sobre la atención primaria en salud (4). Para ello se construyeron y equiparon seis módulos de atención en diferentes localidades ${ }^{(5)}$. Posteriormente, con la intención de brindar una mejor distribución y mayor comodidad a los internos en el proceso de aprendizaje y formación, se implementaron tres nuevos módulos (Tabla 1). El InR forma a los internos de las facultades de Medicina, Enfermería y Odontología, en actividades preventivo-promocionales en familias, comunidades e instituciones; en prevención de riesgos y daños; fomento de estilos de vida saludables; actividades científicas, y atención de morbilidad, siendo esta última la de mayor dedicación. El cumplimiento de los objetivos programados se realiza a través de la supervisión de las labores del equipo de internos a cargo de un equipo de tutores - docentes de la UNSAAC. Para garantizar la sostenibilidad de este programa, desde 1996 la Dirección Regional de Salud Cusco y la UNSAAC apoyan con dinero y traslado, respectivamente, a los estudiantes.

Consideramos que la formación que deja el InR al estudiante de pregrado de la UNSAAC es satisfactoria, y el realizarlo en localidades de extrema pobreza (Tabla 1) le da un valor humano agregado, y garantiza una práctica social acorde con las necesidades de la comunidad, con la participación de la población en forma activa, y tratando los problemas de salud en su ámbito. Con este tipo de programas se ha logrado reorientar la 
formación médica para atender a la comunidad, mejorar el entendimiento de los determinantes sociales y motivar el interés de los estudiantes hacia la APS.

Conflictos de interés: los autores son parte del cuerpo estudiantil y docente de la Universidad Nacional San Antonio Abad del Cusco.

\section{REFERENCIAS BIBLIOGRÁFICAS}

1. Organización Panamericana de la Salud (OPS). La formación en Medicina orientada hacia la atención primaria de salud. Serie: La renovación de la atención primaria de salud en las américas. N. ${ }^{\circ}$. Washington, DC: OPS; 2008.

2. Universidad Peruana Cayetano Heredia (UPCH). Rotación de Internado Interregional Descentralizado (IIRD). Silabo de internos. Lima: UPCH; 2012.

3. Millán KT, Vargas C, Madrid C. Internado rural en la carrera de medicina de la Universidad de Chile; una experiencia de aprendizaje significativo. Educación Médica. 2006;9(3):116-7.

4. Farfán Cantero R, Saco Méndez S. Integración Universidad Servicios de Salud-Comunidad en la Facultad de Medicina Humana de La Universidad Nacional de San Antonio Abad del Cusco. SITUA. 1998;VI(11).

5. Farfán Cantero R. Odisea de la educación médica en Cusco. SITUA. 2002;10(20).

Correspondencia: Roy R. Vásquez Sullca

Dirección: Av. de La Cultura 1906, Cusco, Perú

Teléfono: (051) 984139020

Correoelectrónico:roy_vasquez@hotmail.com

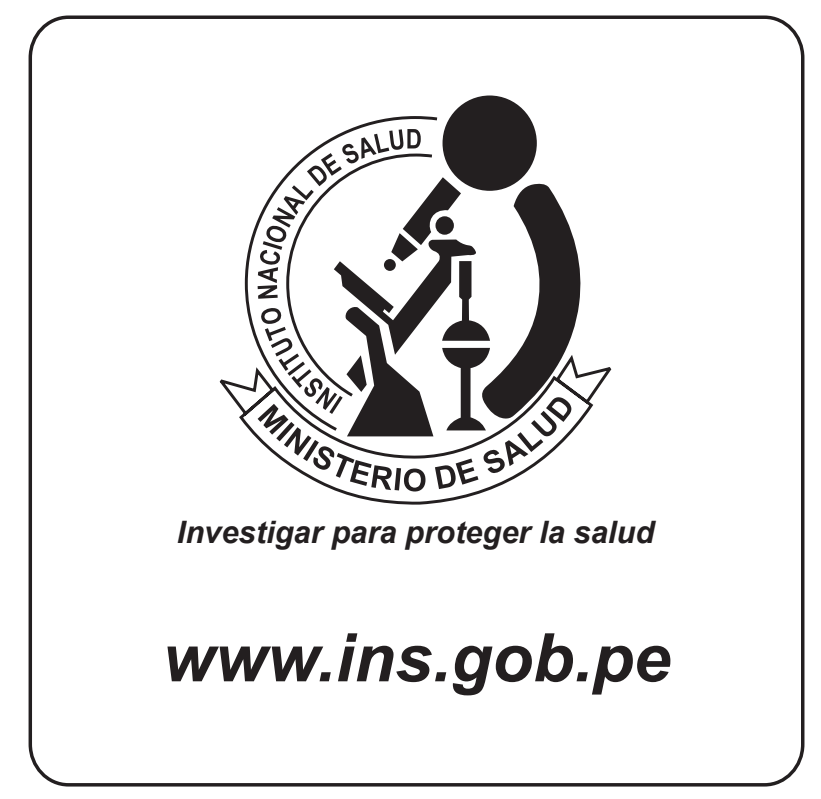

\section{OPINIÓN DE LOS MÉDICOS DE ATENCIÓN PRIMARIA SOBRE EL USO DE UN SISTEMA DE AJUSTE DE RIESGOS: LOS ADJUSTED CLINICAL GROUPS}

\section{PRIMARY CARE DOCTORS' OPINION ON A RISK ADJUSTMENT SYSTEM: ADJUSTED CLINICAL GROUPS}

Antoni Sicras-Mainar ${ }^{1, a}$, Alexandra Prados-Torres
Ruth Navarro-Artieda

Sr. Editor. En el ámbito de la gestión, las experiencias en separar la financiación, la compra y la provisión de servicios requieren de instrumentos muy precisos de evaluación y medida de la actividad asistencial (1). En diferentes países, entre ellos en España (Cataluña), se están desarrollando algunas experiencias en la financiación por cápita como mecanismo para la asignación de los recursos asistenciales en base territorial (2). En este sentido, el sistema Adjusted Clinical Groups (ACG), desarrollado por la Universidad Johns Hopkins, es uno de los sistemas de clasificación de pacientes (SCP; ajuste de riesgos) más ampliamente utilizados a nivel internacional. Permite clasificar grupos de pacientes con similares características clínicas y de consumo de recursos ${ }^{(3)}$. En general, la evidencia que se tiene de la aplicación de los ACG en nuestro medio está estudiada, y su uso se está extendiendo en distintos territorios ${ }^{(4-5)}$. En cambio, no se conoce la opinión que tienen los profesionales médicos de España sobre estos SCP. Este estudio forma parte del proyecto titulado "Factores clínicos como determinantes de la utilización de los recursos sanitarios en Atención Primaria: perfiles de usuarios y diseño de modelos predictivos de utilización, relación entre morbilidad, efectividad y costes, y exploración de estrategias de implantación de sistemas de ajuste por casuística" (PI081567; años 2009-2011), en el que participan diversos centros de las comunidades autonómicas de Cataluña, Aragón y Baleares, financiado por el Fondo de Investigación Sanitaria del Instituto de

\footnotetext{
Dirección de Planificación, Badalona Serveis Assistencials. Barcelona, España.

Instituto Aragonés de las Ciencias de la Salud. Zaragoza, España.

Hospital Germans Trias i Pujol, Barcelona, España.

a Médico doctor en Medicina, especialista en Salud Pública; ${ }^{b}$ médico doctor en Medicina, especialista en Salud Pública; ${ }^{c}$ médico especialista en Documentación Médica

Recibido: 15-02-13 Aprobado: 20-02-13
}

Citar como: Sicras-Mainar A, Prados-Torres A, Navarro-Artieda R. Opinión de los médicos de atención primaria sobre el uso de un sistema de ajuste de riesgos: los adjusted clinical groups [carta]. Rev Peru Med Exp Salud Publica. 2013;30(2):345-7. 